\title{
Cognitive-Affective Status in Anorexia Nervosa: Self-image and Absence of Positive Emotions
}

\author{
Suzanna Lundblad', Jonas Magnusson'1, Berit Hansson ${ }^{1}$ and Trevor Archer ${ }^{2,3^{*}}$ \\ ${ }^{1}$ Sahlgrenska University Hospital, Affectiva II Anorexia \& Bulimia Clinic for Adults, S-41666 Gothenburg, Sweden \\ ${ }^{2}$ University of Gothenburg, Department of Psychology, Gothenburg, Sweden
}

${ }^{3}$ Network for Empowerment and Well-being, Sweden

\begin{abstract}
The relationships between eating disorder patterns (Eating Disorders Examination Questionnaire; EDE-Q), alexithymia (Toronto Alexithymia Scale; TAS) and cognitive-affective (Positive Affect Negative Affect Schedule; PANAS) status in forty-nine patients presenting anorexia (AN) in an age- and education-matched comparison with thirty-four healthy controls were investigated. AN patients expressed higher levels of restraint, eating concern, shape concern, weight concern and a higher global score on the Eating Disorders Examination Questionnaire. These responses were linked to higher scores on Emotional identifying, Emotional describing, Emotional orientation and Total scoring on the Toronto Alexithymia Scale. AN patients expressed also less positive affect (PA) and more negative affect (NA) than the healthy matched controls. Linear regression analysis indicated that three factors predicted the AN condition, namely Shape concern $(\beta=0.689)$ and Weight concern $(\beta=0.570)$ whereas PA was counter-predictive $(\beta=-0.291)$, whereas for the Control condition, only PA was predictive $(\beta=0.350)$. The present findings are discussed from a perspective of documented regional brain dysfunctions underlying cognitive and emotional domains that have been obtained from neuro-imaging studies of anorexia.
\end{abstract}

Keywords: Anorexia; Eating disorder; Alexithymia; Affect healthy controls patients

\section{Introduction}

The anorexia nervosa (AN) condition presents a serious and potentially life-threatening eating disorder (ED) that is characterized by episodes of self-starvation and excessive weight loss that may be accompanied by excessive exercise [1]. The trans-diagnostic model of EDs is a conceptualisation of factors maintaining ED's across different diagnoses (Anorexia Nervosa, Bulimia Nervosa, EDNOS etc.). The model proposes that central to these disorders is a dysfunctional system of self-evaluation of self-worth, where self-worth is determined from perceived shape and weight and also by what extent individuals deem themselves to be in control of their shape and weight. This need for control and a specific body shape and weight is in its turn that which motivates dietary restraint and the consequent operationalization of this into various behavioural strategies and dietary rules that can either be successful (resulting in weight loss) or fail in implementation (subjective or objective binge eating episodes for example, often follow from failure to abide by dietary rules) [2,3]. Support for this model showing the centrality of over-evaluation of weight and shape and that this cognitive mind-set in its turn influences Eating restraint [4]. Studies have also shown that low self-esteem is a general influence on over-evaluation of weight and shape [5]. Since low self-esteem in its turn has been shown to be associated with higher negative affect as measured with Positive Affect and Negative Affect Schedule [6], it would therefore seem conceivable that individuals suffering from ED psychopathology experience less positive and more negative affect, as a consequence of low self-esteem.

The incidence of alexithymia in AN is about $77 \%$ with accompanying emotional problems [7], compared to a $13 \%$ incidence in the general population [8]. Clinical evidence implies that AN patients present major problems with attachment anxiety and negative affect $[9,10]$. The notion of $\mathrm{AN}$ as an anxiety-related disorder seems warranted in view of the evidence implicating anxiety, stress, fear, and avoidance learning factors in the persistence of abnormal eating habits $[11,12]$. It has been observed that AN patients showed significantly greater implicit positive affect toward pleasant images and significantly greater implicit negative affect toward unpleasant, high-calorie food and overweight body type images [13]. Depressiveness among these patients was found to exert much influence too in alexithymia [7], although it did not account for all the cognitive-affective disturbances in the disorder. There appears to be a strong relationship between alexithymia and Intolerance of Uncertainty in AN patients [14]. Regional brain changes were shown to bear some relationship to alexithymia in AN; for example [15] obtained fluctuations in subcallosal cingulate connectivity that correlated with the degree of alexithymia.

The purpose of the present study was to ascertain the relationships between eating disorder patterns, alexithymia and cognitive-affective status in patients presenting $\mathrm{AN}$ in an age- and education-matched comparison with healthy controls.

\section{Method and Materials}

\section{Participants}

Forty-nine female patients (age range: 24 to 42 years) presenting eating disorders at diagnosis, but here limited to those presenting AN, with a history of unsuccessful treatment interventions and referred from the Department of General Psychiatry, Sahlgrenska University Hospital

*Corresponding author: Archer T, Department of Psychology, University of Gothenburg, Box 500, S-405 30 Gothenburg, Sweden, Tel: +46 31 7864694; E-mail: trevor.archer@psy.gu.se

Received: November 02, 2015; Accepted: November 03, 2015; Published: November 15, 2015

Citation: Lundblad S, Magnusson J, Hansson B, Archer T (2015) Cognitive-Affective Status in Anorexia Nervosa: Self-image and Absence of Positive Emotions. Clin Exp Psychol 1: 104. doi:10.4172/cep.1000104

Copyright: (c) 2015 Lundblad S, et al. This is an open-access article distributed under the terms of the Creative Commons Attribution License, which permits unrestricted use, distribution, and reproduction in any medium, provided the original author and source are credited. 
(Gothenburg, Sweden) participated in the study. All the patients were ethnic Scandinavians from higher socioeconomic groups and welleducated. They had all undergone further education at university level, following high-school graduation, for at least three years. They originated from an affluent-level economic status and upper-middle social-family backgrounds. They had all been afflicted with the AN symptoms for over five years at the time of arrival at the Anorexia \& Bulimia Clinic for Adults (Sahlgrenska University Hospital), and described themselves as "well-behaved girls". The thirty-four healthy volunteer participants (i.e. control subjects) were a group of agematched and education-level-matched female subjects.

The ethics protocol of the University Hospital Sahlgrenska was applied and maintained. The regional ethical review board in Gothenburg approved of the study design. All the patients who were contacted agreed to participate $(\mathrm{N}=49)$. Upon arrival at the clinic, each patient described her type of eating disorder and completed the Eating Disorder Inventory-2 to obtain an estimation of eating disorder and each was then given their diagnosis by the presiding staff. Thereafter, each patient was allowed to complete the questionnaire. Specifically, each patient was met on arrival at the clinic by the respective professional workers, i.e. nurse, psychologist, psychiatrist, and after a preliminary discussion was asked whether or not she would be willing to complete a questionnaire in order for the psychiatrist to obtain further insights into the AN condition. Following this, each patient received instructions and was assigned 30-45 min to respond to the items in the instrument.

\section{Instruments}

Background health information: Background variables, such as age, years of education, smoking and drinking habits, pain, use of mood-enhancing drugs and analgesics, and frequency and duration of exercise, etc., were completed according to the description and procedure outlined in [16]

Positive affect negative affect schedule: This is one of the most commonly applied instruments for estimating high arousal affect and was developed on the premise that PA and NA represent two orthogonal independent dimensions: high PA versus low PA and high NA versus low NA [17]. The instrument instructs participants to rate to what extent they have experienced generally the 20 different feelings or emotions (10 PA and $10 \mathrm{NA}$ ) during the last week, using a 5-point Likert scale $(1=$ very slightly, $5=$ extremely). The 10 -item PA scale includes adjectives such as "strong, proud, and interested"; the 10-item NA scale includes adjectives such as "afraid, ashamed, and nervous". In their study, [18] reported a Cronbach's alpha of .88 for the PA scale and .83 for the NA scale. In the present study, the PA and NA scales showed similar internal reliability (.87 for PA and .79 for NA).

Eating Disorders Examination-Questionnaire (EDE-Q): The EDE-Q was originally derived from the Eating disorder examination [19], which is a semi-structured interview method. EDE has long been considered the "gold-standard" for diagnosis and assessment of Eating disorders [20]. EDE-Q was designed to provide a similarly thorough assessment of ED symptoms and behaviours. Studies of the validity of EDE-Q has shown high levels of concordance between EDE and EDE-Q regarding measurement of attitudes central to ED's $[21,22]$. EDE-Q is at present one, if not the most widely used self-report instrument for measuring ED's $[23,24]$. It is composed of 36 items measuring behaviours and experiences relevant for ED's that has taken place during the last 28 days. Four subscales can be calculated: 1) Restraint, 2) Eating concern, 3) Weight concern and 4) Shape concern. Items making up the subscales are answered in a 6-point Likert format. A global score measuring overall severity of the disorder may also be calculated by adding mean scores from the four subscales. Version 6.0 procedures for calculating results were used. Cronbach's alpha values were as follows: Restraint .90, Eating concern .87, Weight concern .87, Shape concern .96 , Global .79.

Toronto alexithymia scale: The Toronto Alexithymia Scale $[25,26]$ offers a self-report instrument that estimates deficiencies in understanding, processing, or describing emotions. The version applied here presents a 20-item (statements) questionnaire on a five-point likert scale. An example of a statement would be: e.g. "When asked which emotion I'm feeling, I frequently don't know the answer", or "I am unsure of which words to use when describing my feelings". The cut-off point for severe alexithymia was 61 points and above. This instrument has been shown to possess a high level of reliability and validity. The TAS-20 has three subscales: (i) Difficulty Describing Feelings subscale is used to measure difficulty describing emotions, (ii) Difficulty Identifying Feeling subscale is used to measure difficulty identifying emotions, and (iii) Externally-Oriented Thinking subscale is used to measure the tendency of individuals to focus their attention externally. The total TAS-20 score shows generally good internal consistency (Cronbach's alpha $=.81$ ) and test-retest reliability $(.77, \mathrm{p}<.01)$. In the present study, Cronbach's alpha was .86; and for Difficulty Describing Feelings .84, Difficulty Identifying Feeling .85, and Externally-Oriented Thinking .69.

\section{Results}

Pillai's MANOVA ( $2 \times 4)$ was applied with AN-patient/Healthy control and Affective personality as independent variables and with EDE-Q-Restraint, -Eating concerns, -Shape concerns, -Weight concerns, -Global score, TAS-20-Total score, -Identifying emotions, -Difficulty describing emotions, -Externally-oriented thoughts, Alexithymia, PANAS-PA and PANAS-NA as dependent variables indicated a significant main effect for AN-patient/Healthy control $(\mathrm{F}(10,67)=2.57, \mathrm{p}<0.02$, eta $=0.269$, observed power $=0.918)$. One-way ANOVA with AN-patient/Healthy control as independent variable indicated significant effects upon EDE-Q-Restraint, -Eating concerns, -Shape concerns, -Weight concerns, -Global score, TAS-20Total score, -Identifying emotions, -Difficulty describing emotions, -Externally-oriented thoughts, Alexithymia, PANAS-PA and PANASNA (as dependent variables). Table 1 presents the means and standard deviations (as well as ANOVA F-values) for participants' responses (AN patients versus Healthy controls) with regard to the dependent variables.

\section{Regression Analysis}

Separate regression analyses were performed on the AN patients and healthy controls in order to ascertain which of the above variables were predictive for either the anorexic or the healthy condition, respectively. The analysis indicated that AN patient condition could be predicted $\left(\mathrm{F}(9,83)=9.14, \mathrm{p}<0.001\right.$, adjusted $\left.\mathrm{R}^{2}=0.625\right)$. The significant predictors were shape concern and weight concern whereas positive affect was counter-predictive (Table 2). Restraint, eating concern, global score, TAS total, TAS-Emotional Identifying, TASEmotional Describing, TAS-External Orientation, and negative affect were nonsignificant. The analysis indicated also that the healthy control condition could be predicted $(\mathrm{F}(9,50)=9.70, \mathrm{p}<0.001$, adjusted $\mathrm{R} 2$ $=0.618$ ). The single significant predictor was positive affect (Table 3 ). Restraint, eating concern, shape concern, weight concern, global score, TAS total, TAS-Emotional Identifying, TAS-Emotional Describing, TAS-External Orientation, and negative affect were non-significant. 


\begin{tabular}{|l|l|l|l|}
\hline Factor & ANOVA & AN patients & Controls \\
\hline EDE-Q-Restraint & $\mathrm{F}(1,104)=53.07^{*}$ & $3.67 \pm 1.72$ & $1.29 \pm 1.46$ \\
\hline EDE-Q-Eating concern & $\mathrm{F}(1,100)=96.31^{*}$ & $3.31 \pm 1.55$ & $0.59 \pm 0.98$ \\
\hline EDE-Q-Shape concern & $\mathrm{F}(1,100)=103.49^{*}$ & $4.78 \pm 1.29$ & $1.82 \pm 1.61$ \\
\hline EDE-Q-Weight concern & $\mathrm{F}(1,97)=55.94^{*}$ & $4.02 \pm 1.58$ & $1.64 \pm 1.48$ \\
\hline EDE-Q-Global score & $\mathrm{F}(1,95)=87.43^{*}$ & $3.92 \pm 1.37$ & $1.34 \pm 1.26$ \\
\hline TAS-Total score & $\mathrm{F}(1,99)=70.92^{*}$ & $57.68 \pm$ & $36.90 \pm 9.53$ \\
\hline $\begin{array}{l}\text { TAS-Emotional } \\
\text { Identifying }\end{array}$ & $\mathrm{F}(1,95)=68.80^{*}$ & $23.17 \pm 6.13$ & $13.07 \pm 4.52$ \\
\hline $\begin{array}{l}\text { TAS-Emotional } \\
\text { Describing }\end{array}$ & $\mathrm{F}(1,95)=56.48^{*}$ & $15.71 \pm 4.45$ & $9.48 \pm 4.17$ \\
\hline TAS-External Orientation & $\mathrm{F}(1,95)=13.57^{*}$ & $18.76 \pm 5.88$ & $14.53 \pm 3.86$ \\
\hline Alexithymia (yes/no $)$ & $\mathrm{F}(1,99)=50.78^{*}$ & $1.12 \pm 0.80$ & $0.15 \pm 0.36$ \\
\hline Positive affect & $\mathrm{F}(1,94)=42.45^{*}$ & $25.01 \pm 8.30$ & $35.98 \pm 7.56$ \\
\hline Negative affect & $\mathrm{F}(1,93)=63.28^{*}$ & $32.72 \pm 8.53$ & $19.62 \pm 5.28$ \\
\hline
\end{tabular}

Values present Means $\pm \mathrm{SD} ;{ }^{*} \mathrm{p}<0.001$

Table 1: Self-reported EDE-Q-Restraint, -Eating concern, -Shape concern, -Weigh concern, -Global score, TAS-Total score. - Problems identifying emotions1, Problems describing emotions2, - Externally-oriented thinking3, Alexithymia, positive and negative affect.

\begin{tabular}{|l|l|l|}
\hline Predictor variable & Standardized $\boldsymbol{\beta}$ & \\
\hline Shape concern & 0.689 & $\mathrm{P}=0.001$ \\
\hline Weight concern & 0.570 & $\mathrm{P}=0.009$ \\
\hline Positive affect & -0.291 & $\mathrm{P}=0.002$ \\
\hline
\end{tabular}

Table 2: Standardized weights from linear regression analysis with $A N$ condition as dependent variable and EDE-Q-Restraint, -Eating concern, -Shape concern, -Weight concern, -Global score, TAS-Total score. - Problems identifying emotions, Problems describing emotions, - Externally-oriented thinking, Alexithymia, positive and negative affect as independent variables.

\begin{tabular}{|l|l|l|}
\hline Predictor variable & Standardized $\boldsymbol{\beta}$ & \\
\hline Positive affect & 0.350 & $\mathrm{P}=0.015$ \\
\hline
\end{tabular}

Table 3: Standardized weights from linear regression analysis with the healthy control condition as dependent variable and EDE-Q-Restraint, -Eating concern, -Shape concern, -Weight concern, -Global score, TAS-Total score. - Problems identifying emotions, Problems describing emotions, - Externally-oriented thinking, Alexithymia, positive and negative affect as independent variables.

\section{Discussion}

Several important aspects associated with eating disorders were presented by the AN patients: they expressed higher levels of restraint, eating concern, shape concern, weight concern and a higher global score on the Eating Disorders Examination Questionnaire (Table 1). These responses were accompanied by higher scores on Emotional identifying, Emotional describing, Emotional orientation and Total scoring on the Toronto Alexithymia Scale, which was confirmed by significantly more affirmative responses to the question regarding alexithymia. AN patients expressed also less PA and more NA than the healthy matched controls. This pattern of results confirms and extends the findings obtained different sets of AN patients [27,28]. In order examine which self-reported attributes may have a predictive relationship to the AN diagnosis a linear regression analysis was performed: three factors predicted the AN condition, namely Shape concern $(\beta=0.689)$ and Weight concern $(\beta=0.570)$ whereas PA was counter-predictive $(\beta=-0.291)$. For the Control condition, only PA was predictive $(\beta=0.350)$. In another study (Garcia et al., under preparation) comparing AN patients with healthy controls (age/education-matched) the former expressed higher scores on past negative and present fatalistic dimensions, conscientiousness, extraversion, agreeableness and neuroticism. Healthy controls scored higher on all other time perspective dimensions and temporal life satisfaction. Hierarchical multiple regressions indicated that AN patients' temporal life satisfaction was predicted by personality although the time perspective dimensions contributed to this prediction to a great extent whereby the unique predictors were past positive and present hedonistic, implying that AN patients' notions of past and present may modulate well-being above and beyond their personal attributes. In a study on well-being among ED patients it was observed that they expressed less PA but not significantly more NA [29], whereas ED-related thoughts seem to be triggered by negative emotions [30,31]. Further, the incidence and negative role of affect and psychological distress in maintaining the disorder in AN patients has been observed [32-34]. Both clinical depression [35] and anxiety [36] are associated markedly with NA, as shown in preclinical studies [37,38]. The markedly higher levels on all five measures of alexithymia (TAS) confirm previous observations [39], and are indicative of negative affect complications [40].

The trans-diagnostic model of EDs, conceptualizing factors considered to maintain the disorders over different diagnoses, proposes that integral to these disorders exists a dysfunctional system pertaining to evaluation of self-worth, wherein self-worth is determined from perceived shape and weight and also by what extent individuals deem themselves to be in control of their shape and weight. The observation that body and weight concerns predicted AN diagnosis (above) implies that cognitive-emotions or selective attentional processes may either contribute to or bolster the disorder. Certainly, any need for control and a specific body shape and weight may in turn motivate dietary restraint and an operationalization into behavioural strategies and dietary rules that can either be successful (resulting in weight loss) or fail in implementation (subjective or objective binge eating episodes) arising, e.g. from failure to abide by dietary rules [41]. The notion of over-evaluation of weight and shape may influence eating restraint compulsivity [42]. It has been observed also that low self-esteem is a general influence on over-evaluation of weight and shape low selfesteem is linked to low PA and high NA [38] and in its turn may be contributory also. AN patients display impairments in several aspects of social cognition $[43,44]$, with specific regard to social signals reflecting emotional states of those in their proximity $[45,46]$ and an exaggerated tendency to observe body rather than eyes [47]. It was found recently that while AN patients reliably orient to different temporal intervals they showed deficits orienting to cue type, or altered selective attention [48]. It has been observed that patients presenting AN display widespread alterations in executive functioning and associated tissue damage [4952]. For example, in a functional magnetic resonance imaging (fMRI) study, reduced activation in the frontostriatal network of AN patients when performing behavioural shifts, independent of the cognitive shift requirement [53]. In an examination of the neural correlates of cognitive flexibility and learning in AN patients, [54] compared 32 adult female AN patients with 32 age-matched female healthy controls undergoing fMRI during the performance of the Wisconsin Card Sorting Task using event-related analysis during the cognitive shift from maintenance of rule-sets and learning. AN patients displayed higher blood-oxygenated-level dependent responses during shifting but lower responses when maintaining rule-sets in comparison with the healthy controls. Posterior cingulated cortex activity was elevated in AN patients but reduced in the healthy controls whereas an opposite pattern was observed in the right precuneus and the caudate nucleus was lower in AN patients. Taken together, it was concluded that the results implied how perserverative tendencies may underlie multiple high-order processes that together contribute to AN.

\section{Limitations}

From a 'trait or state' perspective the current study's sample may 
Citation: Lundblad S, Magnusson J, Hansson B, Archer T (2015) Cognitive-Affective Status in Anorexia Nervosa: Self-image and Absence of Positive Emotions. Clin Exp Psychol 1: 104. doi:10.4172/2471-2701.1000104

be deemed as lacking in generalizability. The large effects of positive and negative affectivity/shape concern and weight concern raises questions regarding state dependent differences between the two groups participating in this study. It is feasible that the present results might to some extent be a product of differing contexts; the participant's experiences of their inner emotional life might have been influenced by the specifics of their situation [55]. The patient sample consisted mostly of newly received patients just having started treatment, but also of patients that already had received psychotherapy for some time. The new patients were asked to participate after having received general information regarding eating disorders, which most certainly meant that many of them were in distress regarding their psychopathology and it's effect on their lives. This distressed state might have led to the participants experiencing and subsequently reporting greater levels of negative feelings. Information received regarding the nature of ED's might also have led to greater awareness of their own preoccupation with body shape and weight. A somewhat similar process might be relevant in the case of the patients who already had started treatment. A psychodynamic psychotherapeutic process (which is the main therapeutic modality these patients were receiving) is to be expected to raise awareness of ones emotional states [56] which might make negative emotions more salient, and arguably also awareness of ones eating disordered behaviours which is the major object of change and discussion in treatment. The control group in contrast was recruited at an evening lecture at a part time course in sexology held at Gothenburg University. The lecture was about an unrelated subject and the participants participated in the study during a break. In this context one could expect the participants from the control group to be both less distressed and less self-aware regarding their self-evaluation of shape and weight.

\section{References}

1. Lundblad S, Garcia D, Hansson B, Archer T (2015) Emotional well-being in anorexia nervosa: negative affect, sleeping problems, use of mood-enhancing drugs and exercise frequency. Arch Depress Anxiety 1:001-005.

2. Fairburn CG, Cooper Z, Shafran R (2003) Cognitive behaviour therapy for eating disorders: A "transdiagnostic" theory and treatment. Behaviour Research and Therapy 41: 509-528.

3. Fairburn CG, Bailey-Straebler S, Basden S, Doll HA, Jones R, et al. (2015) A transdiagnostic comparison of enhanced cognitive behavior therapy (CBT-E) and interpersonal psychotherapy in the treatment of eating disorders. Behav Res Ther 70: 64-71.

4. Hoiles KJ, Egan SJ, Kane RT (2012) The validity of the transdiagnostic cognitive behavioral model of eating disorders in predicting dietary restraint. Eat Behav 13: 123-126

5. Lampard AM, Tasca GA, Balfour L, Bissada H (2013) An evaluation of the transdiagnostic cognitive-behavioral model of eating disorders. Eur Eat Disord Rev. 21:99-107.

6. Palomo T, Beninger RJ, Kostrzewa RM, Archer T (2008) Affective atatus in relation to impulsive, motor and motivational symptoms: personality, development and physical exercise. Neurotox Res 14: 151-168.

7. Torres S, Guerra MP, Lencastre L, Miller K, Vieira FM, et al. (2015) Alexithymia in anorexia nervosa: the mediating role of depression.Psychiatry Res 225: 99-107.

8. Salminen JK, Saarijärvi S, Aärelä E, Toikka T, Kauhanen J (1999) Prevalence of alexithymia and its association with sociodemographic variables in the general population. J Psychosom Res 46: 75-82.

9. Harney MB, Fitzsimmons-Kraft EE, Maldonado CR, Bardone-Cone AM (2014) Negative affective experiences in relation to stages of eating disorder recovery. Eat Behav 15: 24-30.

10. Koizumi M, Takagishi H (2014) The relationship between child maltreatment and emotion recognition. PloS One e86093.
11. Guarda AS, Schreyer CC, Boersma GJ, Tamashiro KL, Moran TH (2015) Anorexia nervosa as a motivated behaviour: anxiety, stress, fear and learning. Physiol Behav pii: S0031-938400202-4.

12. Wable GS, Min JY, Chen YW, Aoki C (2015) Anxiety is correlated with running in adolescent female mice undergoing activity-based anorexia. Behav Neurosci 129: $170-82$

13. Spring VL, Bulik CM (2014) Implicit and explicit affect toward food and weight stimuli in anorexi nervosa. Eat Behav 15: 91-94.

14. Abbate-Daga G, Quaranta M, Marzola E, Amianto F, Fassino S (2015) The relationship between alexithymia and Intolerance of Uncertainty in Anorexia nervosa. BMC Psychiat 48: 202-208.

15. Hayes DJ, Lipsman N, Chen DQ, Woodside DB, Davis KD, et al. (2015) Subcallosal cingulated connectivity in anorexia nervosa patients differs from healthy controls: a multi-tensor tractography study. Brain Stimul 8: 758-768.

16. Palomo T, Beninger RJ, Kostrzewa RM, Archer T (2008) Affective atatus in relation to impulsive, motor and motivational symptoms: personality, development and physical exercise. Neurotox Res 14: 151-168.

17. Watson D, Tellegen A (1985) Towards a consensual structure of mood. Psycho Bull 98: 219-35.

18. Lindahl M, Archer T (2013) Depressive expression and anti-depressive protection in adolescents: Stress, positive affect, motivation and Self-efficacy. Psychology 4:495-505.

19. Fairburn CG, Beglin SJ (1994) Assessment of eating disorders: interview or self-report questionnaire. Int J Eat Disord 16: 363-370.

20. Garner D (2002) Measurement of eating disorder psychopathology. In: CG Fairburn \& KD Brownell (Eds.) Eating Disorders and Obesity: A Comprehensive Handbook ( $2^{\text {nd }}$ Edition), pp. 141-146, New York, Guilford Press.

21. Grilo CM, Masheb RM, Wilson GT (2001) Different methods for assessing the features of eating disorders in patients with binge eating disorder: a replication. Obes Res 9: 418-422.

22. Barnes RD, Masheb RM, White MA, Grilo CM (2011) Comparison of methods for identifying and assessing obese patients with binge eating disorder in primary care settings. Int J Eat Disord 44: 157-163.

23. Mond JM, Hay PJ, Rodgers B, Owen C, Beumont PJV (2104) Validity of the Eating Disorder Examination Questionnaire (EDE-Q) in screening for eating disorders in community samples. Behaviour Research and Therapy 42: 551567 .

24. Berg KC, Peterson CB, Frazier P, Crow SJ (2012) Psychometric evaluation of the eating disorder examination and eating disorder examination-questionnaire: A systematic review of the literature. Int J Eat Disord 45:428-438.

25. Bagby RM, Taylor GJ, Parker JD (1988) Construct validity of the Toronto Alexithymia Scale. Psychother Psychosom 50: 29-34.

26. Taylor GJ, Ryan D, Bagby RM (1985) Toward the development of a new selfreport alexithymia scale. Psychother Psychosom 44: 191-9.

27. Lundblad S, Hansson B, Archer T (2014) Affect-group intervention for alexithymia in eating disorders. Int J Emerg Ment Hum Resourc 17: 219-223.

28. Lundblad S, Garcia D, Hansson B, Archer T (2015) Emotional well-being in anorexia nervosa: negative affect, sleeping problems, use of mood-enhancing drugs and exercise frequency. Arch Depress Anxiety 1: 1-5.

29. Magallares A (2012) Well-being and prejudice toward obese people in women at risk to developing eating disorder. Span J Psychol 15: 1293-302.

30. Haedt-Matt AA, Keel PK (2011) Revisiting the affect regulation model of binge eating: A meta-analysis of studies using ecological momentary assessment Psychol Bull 137: 660-681.

31. Wildes JE, Marcus MD, Bright AC, Dapelo MM, Psychol MC (2012) Emotion and eating disorder symptoms in patients with anorexia nervosa: An experimental study. International Journal of Eating Disorders 45: 876-882.

32. Brand-Gothelf A, Leor S, Apter A, Fennig S (2014) The impact of comorbid depressive and anxiety disorders on severity of anorexia nervosa in adolescent girls. J Nerv Ment Dis 202: 759-762.

33. El Ghoch M, Milanese C, Calugi S, Pellegrini M, Battistini NC, et al. (2014) Bodycomposition, eating disorder psychopathology, and psychological distress in anorexia nervosa: a longitudinal study. Am J Clin Nutr 99: 771-778. 
Citation: Lundblad S, Magnusson J, Hansson B, Archer T (2015) Cognitive-Affective Status in Anorexia Nervosa: Self-image and Absence of Positive Emotions. Clin Exp Psychol 1: 104. doi:10.4172/2471-2701.1000104

Page 5 of 5

34. Engel SG, Wonderlich SA, Crosby RD, Mitchell JE, Crow S, et al. (2013) The role of affect in the maintenance of anorexia nervosa: evidence from a naturalistic assessment of momentary behaviours and emotion. J Abnorm Psychol 122: 709-719.

35. Hughes E (2012) Comorbid depression and anxiety in childhood and adolescent anorexia nervosa: Prevalence and implications for out- come. Clinical Psychologist. 16: 15-24.

36. Swinbourne J, Hunt C, Abbott M, Russell J, St Clare T, et al. (2012) The comorbidity between eating disorders and anxiety disorders. Prevalence in an eating disorder sample and anxiety disorder sample. Australian \& New Zealand Journal of Psychiatry 46: 18-131.

37. Archer T, Adrianson L, Plancak A, Karlsson E (2007) Influence of affective personality upon cognition-mediated emotion-processing: need for empowerment. Eur J Psychiat 21: 248-262.

38. Archer T, Adolfsson B, Karlsson E (2008) Affective personality as cognitiveemotional presymptom profiles regulatory for self-reported health predispositions. Neurotox Res 14: 21-44.

39. Lundblad S, Hansson B, Archer T (2014) Affect-group intervention for alexithymia in eating disorders. Int J Emerg Ment Hum Resourc 17: 219-223.

40. Nekouei ZK, Doost HT, Yousefy A, Manshaee G, Sadeghei M (2014) The relationship of alexithymia with anxiety-depression-stress, quality of life, and social support in coronary heart disease (a psychological model). J Educ Health Promot 3: 68.

41. Fairburn CG, Cooper Z, Shafran R (2003) Cognitive behaviour therapy for eating disorders: A "transdiagnostic" theory and treatment. Behaviour Research and Therapy 41: 509-528.

42. Hoiles KJ, Egan SJ, Kane RT (2012) The validity of the transdiagnostic cognitive behavioral model of eating disorders in predicting dietary restraint. Eat Behav 13: 123-126

43. Caglar-Nazali HP, Corfield F, Cardi V, Ambwani S, Leppanen J, et al. (2014) A systematic review and meta-analysis of 'Systems for Social Processes' in eating disorders. Neurosci Biobehav Rev 42: 55-92.

44. Zucker NL, Losh M, Bulik CM, Labar KS, Piven J, et al. (2007) Anorexia nervosa and autism spectrum disorders: guided investigation of social cognitive endophenotypes. Psychol Bull 133: 976-1006.
45. Cserjesi R, Vermuelen N, Lenard L, Luminet O (2011) Reduced capacity in automatic processing of facial expression in restrictive anorexia nervosa and obesity. Psychiatry Res 188: 253-257.

46. Zhu Y, Hu X, Wang J, Chen J, Guo Q, et al. (2012) Processing of food, body, and emotional stimuli in anorexia nervosa: a systematic review and metaanalysis of functional magnetic resonance studies. Eur Eat Disord Rev 20: $439-450$.

47. Watson KK, Werling DM, Zucker NL, Platt ML (2010) Altered social reward and attention in anorexia nervosa. Front Psychol 1: 36.

48. Dalmaso M, Castelli L, Franchetti L, Carli L, Todisco P, et al. (2015) Altered orienting of attention in anorexia nervosa. Psychiat Res 229: 318-325.

49. Muhlau M, Gaser C, Ilg R, Conrad B, Leibl C, et al. (2007) Gray matter decrease of the anterior cingulated cortex in anorexia nervosa. Am J Psychiat 164: $1850-1857$

50. Sato Y, Saito N, Utsumi A, Aizawa E, Shoji T, et al. (2013) Neural basis impaired cognitive flexibility in patients with anorexia nervosa. Plos One 8: e61108.

51. Shott ME, Filoteo JV, Jappe LM, Pryor T, Maddox WT, et al. (2012) Altered implicit category learning in anorexia nervosa. Neuropsychology 26: 191-201.

52. Titova OE, Hjorth OC, Schioch HB, Brooks SJ (2013) Anorexia nervosa is linked to reduced brain structure in reward and somatosensory regions: a metaanalysis of VBM studies. BMC Psychiatry. 13: 110.

53. Zastrow A, Kaiser S, Stippich C, Walther S, Herzog W, et al. (2009) Neural correlates of impaired cognitive-behavioral flexibility in anorexia nervosa. Am J Psychiat 166: 608-616.

54. Lao-Kaim NP, Fonville L, Glampietro VP, Williams SCR, Simmons A, et al. (2015) Aberrant function of learning and cognitive control networks underlie inefficient cognitive cognitive flexibility in anorexia nervosa: a cross-sectional fMRI study. Plos One 10: e0124027.

55. Kraemer HC, Gullion CM, Rush AJ, Frank E, Kupfer DJ (1994) Can state and trait variables be disentangled? A methodological framework for psychiatric disorders. Psychiatry Research: 52: 55-69.

56. Grabe HJ, Frommer J, Ankerhold A, Ulrich C, Gröger R, et al. (2008) Alexithymia and outcome in psychotherapy. Psychotherapy and Psychosomatics 77: 189194. 